\title{
A Dual-Center Cohort Study on The Association Between Early Deep Sedation and Clinical Outcomes in Mechanically Ventilated Patients During the COVID-19 Pandemic: the COVID-SED Study
}

Robert J. Stephens

Barnes-Jewish Hospital

Erin M. Evans

University of lowa

Michael J. Pajor

Barnes-Jewish Hospital

Ryan D. Pappal

Washington University in St. Louis

Haley M. Egan

University of lowa

Max Wei

University of lowa

Hunter Hayes

University of lowa

Jason A. Morris

Harvard Affiliated Emergency Medicine Residency

Nicholas Becker

Mount Sinai Medical Center

Brian W. Roberts

Cooper University Hospital

Marin H. Kollef

Washington University in St. Louis

Nicholas M. Mohr

University of lowa

Brian M. Fuller ( $\nabla$ fullerb@wustl.edu )

Washington University in St. Louis 
Keywords: COVID, deep sedation, emergency department, mechanical ventilation

Posted Date: March 1st, 2022

DOI: https://doi.org/10.21203/rs.3.rs-1389892/v1

License: (c) (1) This work is licensed under a Creative Commons Attribution 4.0 International License. Read Full License 


\section{Abstract}

Background: Mechanically ventilated patients have experienced greater periods of prolonged deep sedation during the coronavirus disease (COVID-19) pandemic. Multiple studies from the pre-COVID era demonstrate that early deep sedation is associated with worse outcome. Despite this, there is a lack of data on sedation depth and its impact on outcome for mechanically ventilated patients during the COVID19 pandemic. We sought to characterize the emergency department (ED) and intensive care unit (ICU) sedation practices during the COVID-19 pandemic, and to determine if early deep sedation was associated with worse clinical outcomes.

Study Design and Methods: Dual-center, retrospective cohort study conducted over six months (March August, 2020), involving consecutive, mechanically ventilated adults. All sedation-related data during the first 48 hours were collected. Deep sedation was defined as Richmond Agitation-Sedation Scale of -3 to -5 or Riker Sedation-Agitation Scale of $1-3$. To examine impact of early sedation depth on hospital mortality (primary outcome) we used a multivariable logistic regression model. Secondary outcomes included ventilator-, ICU-, and hospital-free days.

Results: 391 patients were studied, and 283 (72.4\%) experienced early deep sedation. Deeply sedated patients received higher cumulative doses of fentanyl, propofol, midazolam, and ketamine when compared to light sedation. Deep sedation patients experienced fewer ventilator-, ICU-, and hospital-free days, and greater mortality ( $30.4 \%$ versus $11.1 \%$ ) when compared to light sedation ( $p<0.01$ for all). After adjusting for confounders, early deep sedation remained significantly associated with higher mortality (adjusted OR $3.44 ; 95 \% \mathrm{Cl} 1.65-7.17 ; \mathrm{p}<0.01$ ). These results were stable in the subgroup of patients with COVID-19.

Conclusions: The management of sedation for mechanically ventilated patients in the ICU has changed during the COVID pandemic. Early deep sedation is common and independently associated with worse clinical outcomes. A protocol-driven approach to sedation, targeting light sedation as early as possible, should continue to remain the default approach.

Clinical Trial Registration: Not applicable.

\section{Introduction}

Approximately $95 \%$ of all critical care trials have failed to demonstrate benefit (1). Despite this, outcomes for the critically ill have improved over the last several decades, owing not to disease- or syndromespecific pharmacotherapies, but secondary to improved supportive routine care. Generated from welldesigned clinical trials and now guideline-supported, some of these routine care practices include lungprotective ventilation with lower tidal volume, conservative fluid management, the use of checklists, and early mobility (2-5). Sedation management is another critical supportive therapy in mechanically ventilated patients. Specifically, a protocol-driven approach, which favors paired spontaneous awakening (SAT) and breathing (SBT) trials, along with light levels of sedation, improves outcome (6-12). The early 
period of respiratory failure [i.e. the emergency department (ED) and first 48 hours of intensive care unit (ICU)] may be especially critical to reduce the overall time spent with periods of deep sedation and coma (13-19).

However, there is little rigorous data on sedation depth and its impact on outcome for mechanically ventilated patients during the coronavirus disease (COVID)-19 era. As an example, a PubMed search (conducted on October 7, 2021) for "COVID-19" yielded 184,897 results; “COVID-19 AND sedation" yielded only 287 , of which only one cohort study examined the impact of sedation depth on outcome $(20,21)$. In a comparison of patients with COVID-19-associated acute respiratory distress syndrome (ARDS) with historical ARDS controls, deep sedation and coma were common and associated with increased mortality (20). High rates of delirium and coma have been observed in critically ill patients with COVID-19 infection (22). Concerns have been raised that surges of COVID-19 cases have impacted the care of critically ill patients without COVID-19 disease, potentially worsening outcomes(23). Overall, these findings suggest that the impact of early deep sedation on outcome during the COVID-19 pandemic, for patients with and without COVID-19, is incompletely understood.

We therefore conducted the COVID-SED Study to: 1) further characterize ED and ICU sedation practices during the COVID-19 pandemic; and 2) test the hypothesis that early deep sedation is associated with worse clinical outcomes.

\section{Methods}

\section{Study Design}

This is a retrospective cohort study conducted over six months (March - August, 2020), involving consecutive adult mechanically ventilated patients at two academic tertiary referral centers. The study is reported in accordance with the Strengthening Reporting of Observational Studies in Epidemiology (STROBE) Statement. (Additional File 1) (24).

The Institutional Review Board (IRB) and Human Research Protection Office (HRPO) at each site approved the study with waiver of informed consent prior to study initiation (IRB \# 202009119 and 202009604).

\section{Participants}

All consecutive mechanically ventilated adult patients admitted to the ICU from the ED were screened via established electronic screening procedures. Inclusion criterion: 1) age $\geq 18$ years; and 2) receipt of mechanical ventilation via an endotracheal tube. In addition to mechanically ventilated patients admitted from the ED, all other mechanically ventilated COVID-19 patients admitted to the intensive care unit were screened for inclusion. This was done to capture all patients with COVID-19 during the six-month enrollment period, provided they satisfied all other inclusion and exclusion criteria. Exclusion criteria targeted patients in whom duration of mechanical ventilation was unlikely to be altered by sedation 
management or those in whom acute injury could act as a confounder with sedation depth: 1) death or transition to comfort measures within 24 hours; 2) acute neurologic injury (e.g. stroke, intracranial hemorrhage, traumatic brain injury, cardiac arrest with residual neurologic deficit, status epilepticus, drug overdose, fulminant hepatic failure); 3) transfer to another hospital; 4) chronic/home ventilation; 5) direct admission to the operating room (OR) from the ED; and 6) extubation in the ED.

\section{Assessments and Outcome Measures}

Clinical variables and outcome measures were objective to ensure ease of abstraction from the electronic medical record. Data were collected and entered into a database with Research Electronic Data Capture (REDCap) tools $(25,26)$. Team members were trained regarding data abstraction. Data quality checks were performed with manual and automated methods, and by enforcing plausible data ranges in the REDCap fields. Prior to analysis, the database was screened for implausible values and the electronic medical record was used to recheck any flagged data.

Baseline data including age, gender, weight, race, comorbid medical conditions, COVID-19 status, vital signs, laboratory values, indication for mechanical ventilation, and ventilator settings were recorded. Process of care variables included ED length of stay, antibiotic use, and vasopressor use. Illness severity was assessed with the modified sequential organ failure assessment (SOFA) score $(27,28)$.

Sedation-related data included induction agents and neuromuscular blockers used for endotracheal intubation. Analgesia- and sedation-related data from the ED and during the first 48 hours of ICU admission included opiates, propofol, benzodiazepines, dexmedetomidine, ketamine, haloperidol, quetiapine, gabapentin, and neuromuscular blockers (i.e. rocuronium, vecuronium, and cisatricurium).

Sedation depth was monitored and recorded according to standard routine care at each site, and included the Richmond Agitation-Sedation Scale (RASS) and the Riker Sedation-Agitation Scale (SAS). Deep sedation was defined as: 1$)$ median RASS of -3 to -5 ; or 2$)$ median SAS of $1-3(15-17,29)$ during the first 48 hours of care from admission to the ICU. This period of early sedation was chosen for several reasons. First, early sedation depth is appears to be an important contributor to outcome in mechanically ventilated patients, as demonstrated by several studies which found deep sedation during the initial 48 hours of mechanical ventilation to be associated with increased mechanical ventilation duration, mortality, incidence of delirium, and longer lengths of stay $(14-16,19)$. Second, this endpoint would allow for an account of the time spent in the ED, which has not been reported before during the COVID-19 pandemic.

Patients were followed until death or hospital discharge. The primary outcome was hospital mortality. Secondary outcomes include ventilator-, ICU-, and hospital-free days.

\section{Statistical Analysis}

Descriptive statistics and frequency distributions were used to assess baseline patient characteristics and sedation-related data according to sedation depth. Categorical data were compared with the chi- 
square test, and continuous data were compared using the independent samples t-test or Mann-Whitney U test after testing for normality of data. Time (in days) to mortality was assessed with the Kaplan-Meier survival estimate and log-rank test, comparing the early deep sedation and light sedation groups. A second Kaplan-Meier survival estimate was also calculated, which also included patients deeply sedated throughout the first week of ICU care.

To examine the impact of early sedation depth on hospital mortality, a multivariable logistic regression model was used, following recommendations that covariates be selected a priori (30). The model was adjusted for covariates previously associated with mortality in this cohort: 1) early deep sedation; 2) age; 3) illness severity; 4) indication for mechanical ventilation; and 5) COVID-19 status. All tests were twotailed and a $p$ value of $<0.05$ was considered statistically significant.

A post-hoc exploratory analysis was conducted after noting a significantly higher proportion of deeply sedated COVID-19 patients (Table 1). Taking a similar approach to the primary analysis, this secondary analysis analyzed and reported the baseline characteristics and sedation-related data according to COVID-19 status. To further explore if deep sedation remained independently associated with worse clinical outcomes, a separate multivariable model was conducted on patients positive for COVID-19. 
Table 1

Characteristics of mechanically ventilated patients based on early sedation depth status.

\section{Early Sedation Depth Status}

\begin{tabular}{llll|} 
Baseline characteristics & $\begin{array}{l}\text { Light Sedation } \\
(\mathbf{n}=108)\end{array}$ & $\begin{array}{l}\text { Deep Sedation } \\
(\mathbf{n}=\mathbf{2 8 3})\end{array}$ & P value \\
\hline Age $(\mathrm{yr})$ & $55.2(19.4)$ & $56.4(16.6)$ & 0.53 \\
Gender & $65(60.2)$ & $169(59.7)$ & 0.93 \\
Male, $\mathrm{n}(\%)$ & $43(39.8)$ & $114(40.3)$ & \\
Female, $\mathrm{n}(\%)$ & & & \\
Body mass index $\left(\mathrm{kg} / \mathrm{m}^{2}\right)$ & $29.5(8.6)$ & $30.0(9.6)$ & 0.61 \\
\hline Race, $\mathrm{n}(\%)$ & $42(38.9)$ & $138(48.8)$ & 0.48 \\
White & $58(53.7)$ & $120(42.4)$ & \\
Black & $3(2.8)$ & $10(3.5)$ & \\
Hispanic & $1(0.9)$ & $4(1.4)$ & \\
Asian & $0(0.0)$ & $1(0.3)$ & \\
Native American & $4(3.7)$ & $10(3.5)$ & \\
Other & & & \\
\hline
\end{tabular}

CHF: congestive heart failure; ESRD: end-stage renal disease; COPD: chronic obstructive pulmonary disease; SOFA: sequential organ failure assessment score; PEEP: positive end-expiratory pressure; ED: emergency department

Continuous variables are reported as mean (standard deviation) and median (interquartile range).

* schizophrenia, bipolar disorder, major depression, anxiety

**modified score, which excludes Glasgow Coma Scale 


\section{Early Sedation Depth Status}

\begin{tabular}{|c|c|c|c|}
\hline Comorbidities, n (\%) & $8(7.4)$ & $28(9.9)$ & 0.45 \\
\hline Dementia & $30(27.8)$ & $106(37.5)$ & 0.07 \\
\hline Diabetes mellitus & $6(5.6)$ & $13(4.6)$ & 0.69 \\
\hline Cirrhosis & $15(13.9)$ & $51(18.0)$ & 0.33 \\
\hline $\mathrm{CHF}$ & $9(8.3)$ & $20(7.1)$ & 0.67 \\
\hline ESRD/Dialysis & $18(16.7)$ & $52(18.4)$ & 0.69 \\
\hline COPD & $4(3.7)$ & $18(6.4)$ & 0.31 \\
\hline Immunosuppression & $11(10.2)$ & $36(12.7)$ & 0.49 \\
\hline Malignancy & $16(14.8)$ & $27(9.5)$ & 0.14 \\
\hline Alcohol abuse & $37(34.3)$ & $83(29.3)$ & 0.35 \\
\hline \multicolumn{4}{|l|}{ Psychiatric* } \\
\hline Positive for COVID-19 & $44(40.7)$ & $159(56.2)$ & 0.01 \\
\hline Temperature (Celsius) & $36.9(1.3)$ & $37.0(1.4)$ & 0.31 \\
\hline Blood pressure $(\mathrm{mmHg})$ & $132.7(34.4)$ & $128.0(29.8)$ & 0.19 \\
\hline Systolic & $82.0(24.2)$ & $79.5(21.6)$ & 0.33 \\
\hline \multicolumn{4}{|l|}{ Diastolic } \\
\hline Lactate (mmol/L) & $2.0(1.3-3.1)$ & $2.1(1.3-3.4)$ & 0.61 \\
\hline Creatinine $(\mathrm{mg} / \mathrm{dl})$ & $1.1(0.8-1.8)$ & $1.2(0.9-2.3)$ & 0.10 \\
\hline Hemoglobin (g/dl) & $12.4(2.5)$ & $12.4(2.5)$ & 0.85 \\
\hline $\mathrm{pH}$ & $7.30(0.12)$ & $7.30(0.12)$ & 0.95 \\
\hline $\mathrm{PaO} 2$ & $137.0(70.9)$ & $121.0(76.9)$ & 0.23 \\
\hline $\mathrm{PaO2}: \mathrm{FiO} 2$ & $241.3(161.0)$ & $184.8(148.3)$ & 0.04 \\
\hline $\mathrm{PaCO} 2$ & $49.4(16.9)$ & $48.7(19.9)$ & 0.77 \\
\hline
\end{tabular}

CHF: congestive heart failure; ESRD: end-stage renal disease; COPD: chronic obstructive pulmonary disease; SOFA: sequential organ failure assessment score; PEEP: positive end-expiratory pressure; ED: emergency department

Continuous variables are reported as mean (standard deviation) and median (interquartile range).

* schizophrenia, bipolar disorder, major depression, anxiety

**modified score, which excludes Glasgow Coma Scale 


\section{Early Sedation Depth Status}

\begin{tabular}{|c|c|c|c|}
\hline SOFA** & $4.5(2.6)$ & $5.3(2.5)$ & 0.01 \\
\hline Reason for mechanical ventilation, $\mathrm{n}(\%)$ & $14(13.0)$ & $44(15.5)$ & 0.01 \\
\hline Sepsis & $18(16.7)$ & $23(8.1)$ & \\
\hline Trauma & $17(15.7)$ & $48(17.0)$ & \\
\hline COPD & $12(11.1)$ & $12(4.2)$ & \\
\hline Drug overdose & $10(9.3)$ & $22(7.8)$ & \\
\hline CHF/pulmonary edema & $13(12.0)$ & $76(26.9)$ & \\
\hline Other & $4(3.7)$ & $10(3.5)$ & \\
\hline Cardiac arrest & $10(9.3)$ & $21(7.4)$ & \\
\hline Altered mental status & $1(0.9)$ & $5(1.8)$ & \\
\hline Angioedema & $1(0.5)$ & $0(0.0)$ & \\
\hline Neuromuscular weakness & $8(7.4)$ & $22(7.8)$ & \\
\hline \multicolumn{4}{|l|}{ Airway protection } \\
\hline Tidal volume (mL/kg PBW) & $6.6(6.1-7.3)$ & $6.5(6.0-7.3)$ & 0.24 \\
\hline PEEP (cm H20) & $6.5(5.0-10.0)$ & $8.0(5.0-12.0)$ & $<0.01$ \\
\hline Fraction of inspired oxygen (\%) & $64.8(25.9)$ & $74.9(26.2)$ & $<0.01$ \\
\hline \multicolumn{4}{|l|}{ Process of Care Variables } \\
\hline ED length of stay (hours) & $5.9(3.8-8.3)$ & $4.0(2.5-6.1)$ & $<0.01$ \\
\hline Antibiotics for infection, $\mathrm{n}(\%)$ & $50(47.6)$ & $119(44.2)$ & 0.56 \\
\hline Vasopressor infusion, n (\%) & $26(24.3)$ & $72(25.9)$ & 0.75 \\
\hline \multicolumn{4}{|c|}{$\begin{array}{l}\text { CHF: congestive heart failure; ESRD: end-stage renal disease; COPD: chronic obstructive pulmonary } \\
\text { disease; SOFA: sequential organ failure assessment score; PEEP: positive end-expiratory pressure; ED } \\
\text { emergency department }\end{array}$} \\
\hline \multicolumn{4}{|c|}{ Continuous variables are reported as mean (standard deviation) and median (interquartile range). } \\
\hline \multicolumn{4}{|c|}{ * schizophrenia, bipolar disorder, major depression, anxiety } \\
\hline **modified score, which excludes Glasgo & scale & & \\
\hline
\end{tabular}

From prior work regarding the impact of early deep sedation on outcome, we estimated that approximately two-thirds of the cohort would experience early deep sedation, with a mortality of $25 \%$ in the early deep sedation group versus $10 \%$ in the light sedation group (19). For $80 \%$ power and alpha of 0.05, we estimated a sample size of 219 (82 light sedation, 137 deep sedation) would be required. Based 
on our prior work regarding mechanically ventilated patients at each site, we were confident that a sixmonth enrollment window would be sufficient to accrue the necessary sample size $(17,18,31-34)$.

\section{Results}

The data presented here was from the first six months of the COVID-19 pandemic and we recognize that practices have evolved dramatically since March of 2020 .

\section{Study Population}

Eight hundred eighty-one patients were assessed for eligibility, and 391 comprised the final study population (Fig. 1). Baseline characteristics according to early sedation depth status are in Table 1. Deeply sedated patients had a higher proportion of patients with COVID-19, and a lower partial pressure of arterial oxygenation to fraction of inspired oxygen ratio (PaO2:FiO2).

\section{Medications Administered}

Medications used for endotracheal intubation are located in Additional Table 1. Sedation variables for the 244 patients that were mechanically ventilated in the ED are in Additional Table 2. ICU sedation variables for the first 48 hours of admission are in Table 2. Deeply sedated patients received higher cumulative doses of fentanyl, propofol, midazolam, hydromorphone, and ketamine when compared to the light sedation group. In addition, deeply sedated patients received neuromuscular blockers more frequently $(41.4 \%$ vs. $2.1 \%, p<0.01)$. 
Table 2

Sedation variables in the intensive care unit during the first 48 hours of admission, according to sedation depth

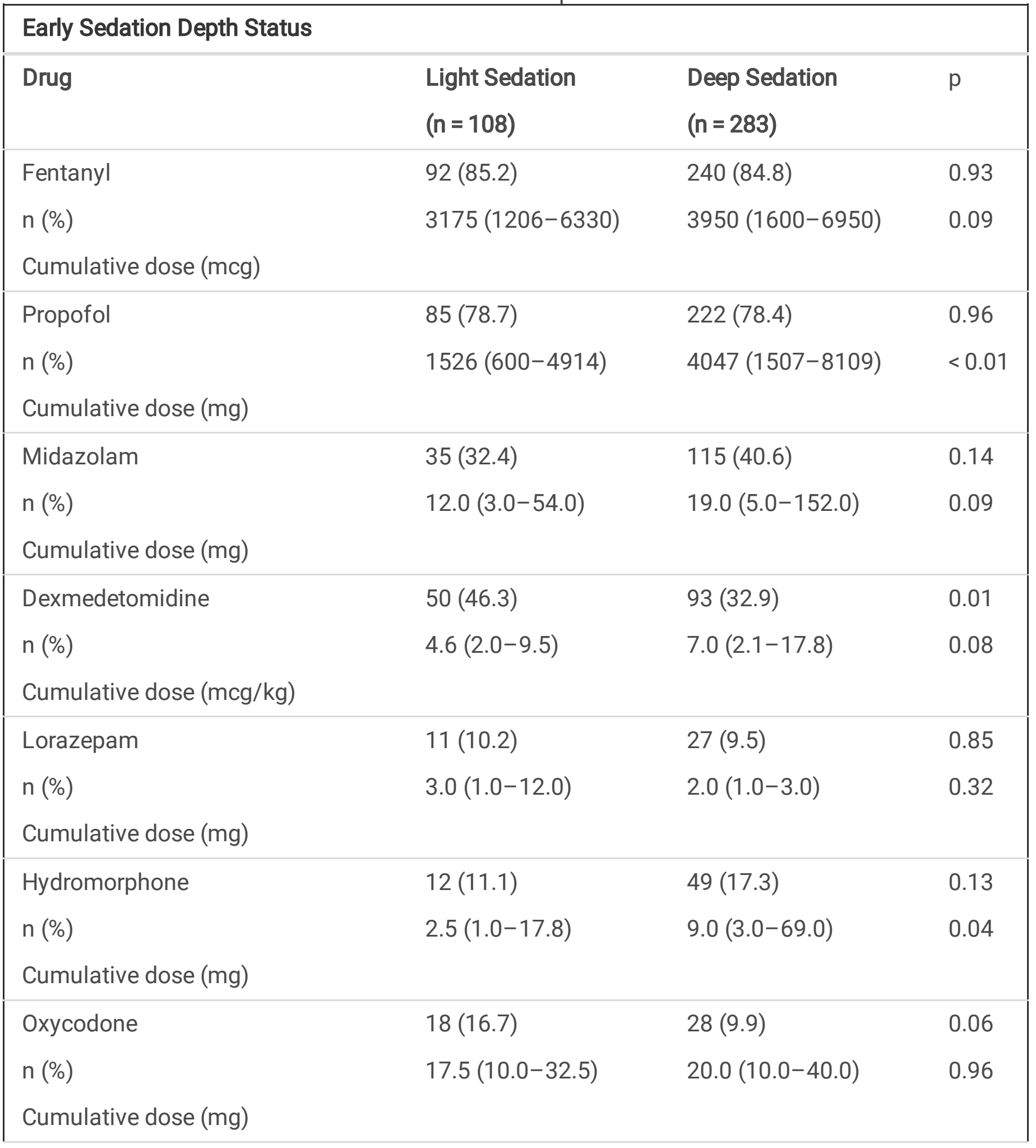

ICU = intensive care unit, RASS = Richmond Agitation-Sedation Scale.

*Denominator is 314 (238 deep sedation group and 76 light sedation group). 


\begin{tabular}{|c|c|c|c|}
\hline \multicolumn{4}{|l|}{ Early Sedation Depth Status } \\
\hline Morphine & $1(0.9)$ & $7(2.5)$ & 0.33 \\
\hline$n(\%)$ & $2.0(\mathrm{NA})$ & $6.5(2.0-12.8)$ & 0.57 \\
\hline \multicolumn{4}{|l|}{ Cumulative dose (mg) } \\
\hline Ketamine & $10(9.3)$ & $38(13.4)$ & 0.26 \\
\hline$n(\%)$ & $87.5(50.0-250.0)$ & $675.0(187.5-2050.0)$ & $<0.01$ \\
\hline \multicolumn{4}{|l|}{ Cumulative dose (mg) } \\
\hline Haloperidol & $9(8.3)$ & $13(4.6)$ & 0.15 \\
\hline$n(\%)$ & $5.0(5.0-10.0)$ & $5.0(5.0-10.0)$ & 0.95 \\
\hline \multicolumn{4}{|l|}{ Cumulative dose (mg) } \\
\hline Quetiapine & $4(3.7)$ & $12(4.2)$ & 0.81 \\
\hline$n(\%)$ & $37.5(25.0-237.5)$ & $200.0(31.3-287.5)$ & 0.91 \\
\hline \multicolumn{4}{|l|}{ Cumulative dose (mg) } \\
\hline Gabapentin & $11(10.2)$ & $11(3.9)$ & 0.02 \\
\hline$n(\%)$ & $600.0(300.0-2100.0)$ & $1200(300-2100)$ & 0.33 \\
\hline \multicolumn{4}{|l|}{ Cumulative dose (mg) } \\
\hline Neuromuscular blocker, n (\%) & $4(2.1)$ & $84(41.4)$ & $<0.01$ \\
\hline RASS Level ICU Day 1 & $-1(-2$ to -0$)$ & $-3(-4$ to -2$)$ & $<0.01$ \\
\hline SAS Level ICU Day 1 & $4(4-4)$ & $3(2-4)$ & $<0.01$ \\
\hline RASS Level ICU Day 2 & $-1(-2$ to 0$)$ & $-3(-5$ to -2$)$ & $<0.01$ \\
\hline SAS Level ICU Day 2 & $4(4-4)$ & $3(3-4)$ & $<0.01$ \\
\hline RASS Level ICU Days 3-7 & $-1(-2$ to 0$)$ & $-3(-4$ to -1$)$ & $<0.01$ \\
\hline SAS Level ICU Days 3-7 & $4(4-4)$ & $3(3-4)$ & $<0.01$ \\
\hline Deep sedation ICU Days 3-7, n (\%)* & $14(18.4)$ & $128(53.8)$ & $<0.01$ \\
\hline Deep sedation until death, $\mathrm{n}(\%)$ & $0(0.0)$ & $94(33.2)$ & $<0.01$ \\
\hline \multicolumn{4}{|c|}{ ICU = intensive care unit, RASS = Richmond Agitation-Sedation Scale. } \\
\hline
\end{tabular}


Deep sedation occurred in $72.4 \%$ of all patients (both COVID-19 and non-COVID-19 cohorts) during the first 48 hours. Sedation levels differed significantly ( $p<0.01$ for each) between the deep sedation and light sedation groups during this period. This difference persisted through the first seven days of mechanical ventilation (Table 2), such that 128 (53.8\%) patients in the deep sedation group experienced deep sedation during the first week of ICU care, as compared to 14 (18.4\%) patients in the light sedation group, $p<0.01$. Ninety-four (33.2\%) deeply sedated patients remained deeply sedated until death, compared to $0(0.0 \%)$ patients in the light sedation group, $p<0.01$.

\section{Subgroup Analyses}

Baseline characteristics according to COVID-19 status are in Additional Table 3. ED sedation variables are in Additional Table 4, and ICU sedation variables from the first 48 hours are in Table 3. No significant differences in medication doses were observed in the ED. In the ICU, COVID-19 patients received significantly higher cumulative doses of fentanyl, propofol, midazolam, hydromorphone, and ketamine when compared to non-COVID patients. COVID-19 patients also received neuromuscular blockers more frequently than non-COVID patients in the ICU $(41.4 \%$ vs. $2.1 \%, p<0.01)$. COVID-19 patients experienced deep sedation more frequently early and throughout the first week of ICU care ( $p<0.01$ for all). Seventyeight (38.4\%) COVID patients remained deeply sedated until death, compared to $16(8.5 \%)$ non-COVID patients. 
Table 3

Sedation variables in the intensive care unit during the first 48 hours of admission, according to COVID status.

\section{COVID Status}

\begin{tabular}{|c|c|c|c|}
\hline Drug & $\begin{array}{l}\text { Non-COVID } \\
(n=188)\end{array}$ & $\begin{array}{l}\text { COVID } \\
(n=203)\end{array}$ & $\mathrm{p}$ \\
\hline Fentanyl & $148(78.7)$ & $184(90.6)$ & $<0.01$ \\
\hline$n(\%)$ & $1562(509-4063)$ & $5350(3275-8050)$ & $<0.01$ \\
\hline \multicolumn{4}{|l|}{ Cumulative dose (mcg) } \\
\hline Propofol & $143(76.1)$ & $164(80.8)$ & 0.26 \\
\hline n (\%) & $2324(1021-6443)$ & 4047 (1227-8127) & 0.02 \\
\hline \multicolumn{4}{|l|}{ Cumulative dose (mg) } \\
\hline Midazolam & $36(19.1)$ & $114(56.2)$ & $<0.01$ \\
\hline$n(\%)$ & $4.0(2.0-30.0)$ & $31.5(5.0-155.0)$ & $<0.01$ \\
\hline \multicolumn{4}{|l|}{ Cumulative dose (mg) } \\
\hline Dexmedetomidine & $91(48.4)$ & $52(25.6)$ & $<0.01$ \\
\hline$n(\%)$ & $5.3(2.2-15.4)$ & $5.3(1.6-13.1)$ & 0.67 \\
\hline \multicolumn{4}{|c|}{ Cumulative dose (mcg/kg) } \\
\hline Lorazepam & $17(9.0)$ & $21(10.3)$ & 0.66 \\
\hline n (\%) & $2.0(1.5-11.0)$ & $2.0(1.0-3.5)$ & 0.37 \\
\hline \multicolumn{4}{|l|}{ Cumulative dose (mg) } \\
\hline Hydromorphone & $38(20.2)$ & $23(11.3)$ & 0.02 \\
\hline n (\%) & $4.5(2.0-11.0)$ & $71.0(9.0-108.0)$ & $<0.01$ \\
\hline \multicolumn{4}{|l|}{ Cumulative dose (mg) } \\
\hline Oxycodone & $28(14.9)$ & $18(8.9)$ & 0.07 \\
\hline n (\%) & $17.5(10.0-37.5)$ & $20.0(10.0-35.0)$ & 0.76 \\
\hline Cumulative dose (mg) & & & \\
\hline
\end{tabular}

ICU = intensive care unit, RASS $=$ Richmond Agitation-Sedation Scale .

*Denominator is 359 (193 COVID group and 166 non-COVID group).

**Denominator is 314 (185 COVID group and 129 non-COVID group). 


\begin{tabular}{|c|c|c|c|}
\hline \multicolumn{4}{|l|}{ COVID Status } \\
\hline Morphine & $1(0.5)$ & $7(3.4)$ & 0.04 \\
\hline$n(\%)$ & $8.0(\mathrm{NA})$ & $3.5(2.0-12.8)$ & 0.86 \\
\hline \multicolumn{4}{|l|}{ Cumulative dose (mg) } \\
\hline Ketamine & $16(8.5)$ & $32(15.8)$ & 0.03 \\
\hline n (\%) & $92.5(50.0-350.0)$ & $950.0(234.0-2050.0)$ & $<0.01$ \\
\hline \multicolumn{4}{|l|}{ Cumulative dose (mg) } \\
\hline Haloperidol & $14(7.4)$ & $8(3.9)$ & 0.13 \\
\hline n (\%) & $5.0(5.0-11.3)$ & $5.0(5.0-8.8)$ & 0.37 \\
\hline \multicolumn{4}{|l|}{ Cumulative dose (mg) } \\
\hline Quetiapine & $5(2.7)$ & $11(5.4)$ & 0.17 \\
\hline n (\%) & $50.0(37.5-300.0)$ & $200.0(25.0-250.0)$ & 0.91 \\
\hline \multicolumn{4}{|l|}{ Cumulative dose (mg) } \\
\hline Gabapentin & $13(6.9)$ & $9(4.4)$ & 0.29 \\
\hline n (\%) & $600.0(300.0-2100.0)$ & $800.0(350.0-2100.0)$ & 0.85 \\
\hline \multicolumn{4}{|l|}{ Cumulative dose (mg) } \\
\hline $\begin{array}{l}\text { Neuromuscular blocker } \\
\text { n (\%) }\end{array}$ & $4(2.1)$ & $84(41.4)$ & $<0.01$ \\
\hline RASS Level ICU Day 1 & $-2(-3$ to -1$)$ & $-3(-4$ to -2$)$ & $<0.01$ \\
\hline SAS Level ICU Day 1 & $3(3-4)$ & $2(1-4)$ & $<0.01$ \\
\hline RASS Level ICU Day 2 & $-1(-2$ to 0$)$ & $-3(-5$ to -2$)$ & $<0.01$ \\
\hline SAS Level ICU Day 2 & $4(3-4)$ & $3(1-3)$ & $<0.01$ \\
\hline RASS Level ICU Days 3-7 & $0(-2$ to 0$)$ & $-3(-5$ to -2$)$ & $<0.01$ \\
\hline SAS Level ICU Days 3-7 & $4(3-4)$ & $2(1-3)$ & $<0.01$ \\
\hline
\end{tabular}

ICU = intensive care unit, RASS = Richmond Agitation-Sedation Scale.

*Denominator is 359 (193 COVID group and 166 non-COVID group).

**Denominator is 314 (185 COVID group and 129 non-COVID group). 


\begin{tabular}{|llll|}
\hline \multicolumn{4}{|l|}{ COVID Status } \\
\hline Deep sedation ICU Day 1, n (\%) & $73(38.8)$ & $118(58.1)$ & $<0.01$ \\
Deep sedation ICU Day 2, n (\%)* & $43(25.9)$ & $117(60.6)$ & $<0.01$ \\
Deep sedation ICU Days 3-7, n (\%)** & $25(19.4)$ & $109(61.6)$ & $<0.01$ \\
Deep sedation until death, $\mathrm{n}(\%)$ & $16(8.5)$ & $78(38.4)$ & $<0.01$ \\
\hline ICU = intensive care unit, RASS = Richmond Agitation-Sedation Scale. & \\
\hline *Denominator is 359 (193 COVID group and 166 non-COVID group). & \\
\hline **Denominator is 314 (185 COVID group and 129 non-COVID group). & \\
\hline
\end{tabular}

Table 4

Unadjusted analysis of clinical outcomes according to early sedation depth.

\begin{tabular}{|lllll|}
\hline Outcome & $\begin{array}{l}\text { Light sedation }(\mathbf{n}= \\
\mathbf{1 0 8})\end{array}$ & $\begin{array}{l}\text { Deep sedation }(\mathrm{n}= \\
\mathbf{2 8 3})\end{array}$ & $\begin{array}{l}\text { OR or Between-Group } \\
\text { Difference } \\
(\mathbf{9 5 \%} \mathrm{Cl})\end{array}$ & $\mathbf{p}$ \\
\hline $\begin{array}{l}\text { Ventilator-free } \\
\text { days }\end{array}$ & $20.7(9.6)$ & $14.7(11.4)$ & $6.04(3.60-8.48)$ & 0.01 \\
\hline $\begin{array}{l}\text { ICU-free days } \\
\text { Hospital-free }\end{array}$ & $18.3(9.9)$ & $12.1(11.0)$ & $6.20(3.82-8.57)$ & 0.01 \\
\hline $\begin{array}{l}\text { Mays } \\
\text { Mortality, } n(\%)\end{array}$ & $12(11.1)$ & $8.0(9.6)$ & $5.74(3.56-7.92)$ & 0.01 \\
\hline ICU = intensive care unit; OR: odds ratio; Cl: confidence interval & & $3.49(1.82-6.70)$ & $<$ \\
\hline
\end{tabular}

\section{Clinical Outcomes}

Table 4 shows that in the unadjusted analysis of clinical outcomes according to sedation depth, deep sedation patients experienced fewer ventilator-, ICU-, and hospital-free days, and greater mortality (30.4\% versus $11.1 \%$ ) when compared to light sedation ( $p<0.01$ for all). On Kaplan-Meier analysis, survival diverged significantly between the early deep sedation and light sedation groups (log-rank $p<0.01$, Fig. 2). After adjusting for confounders (Additional Table 5), early deep sedation remained significantly associated with higher mortality (adjusted OR 3.44; 95\% Cl 1.65-7.17; $p<0.01$ ).

In the subgroup analysis (Additional Table 6), similar unadjusted clinical outcomes according to COVID status were seen, such that COVID patients experienced fewer ventilator-, ICU-, and hospital-free days ( $p<$ 0.01 for all). Mortality was $41.4 \%$ in COVID patients versus $7.4 \%$ in non-COVID patients $(p<0.01)$. After adjusting for confounders (Additional Table 6), early deep sedation remained significantly associated 
with higher mortality (adjusted OR 2.76; 95\% $\mathrm{Cl} 1.26-6.06 ; \mathrm{p}<0.01$ ), though illness severity remained an important variable in this analysis.

\section{Discussion}

Given the importance of high-quality supportive therapies in critical illness, the potential impact of early sedation depth on clinical outcomes, and a dearth of early sedation data in the COVID-19 era, we conducted the COVID-SED study to characterize ED and early ICU sedation practices during the COVID-19 pandemic and assess the impact of early deep sedation on clinical outcomes. We found that over $70 \%$ of mechanically ventilated patients experienced early deep sedation, with significant differences in cumulative medication doses and neuromuscular blockade. In addition, early deep sedation frequently persisted throughout the first week of mechanical ventilation and was negatively associated with outcome.

Our most important finding was an association between early deep sedation and worse clinical outcomes. Early deep sedation was associated with fewer ventilator-, ICU-, and hospital-free days, and increased hospital mortality. These results remained significant after adjustment for confounders, and were consistent in the subgroup of patients with COVID. Our findings are supported by prior work in the pre-COVID era, which showed the negative relationship between early deep sedation and patient-centered clinical outcomes (14-19). Additionally, these findings are congruent with a recent analysis that examined the impact of deep sedation in a comparison of patients with COVID-associated ARDS with non-COVID historical controls (20). The findings of the COVID-SED Study are further support of a guideline- and protocol-driven approach to sedation management, regardless of COVID status (35).

A second important finding is the characterization of sedation practices during the first wave of the COVID pandemic. Sedation in the ED was similar to prior work, suggesting that the COVID era influenced ED-based sedation little (17). However, compared with pre-COVID work, sedation in the ICU saw an increased use and higher doses of fentanyl, benzodiazepines, and ketamine, which appeared largely driven by COVID status (17). The occurrence rate of $72.4 \%$ of early deep sedation is also higher than that seen in recent pre-COVID publications and further highlights the rapidly-adopted changes in sedation practice that occurred with the COVID pandemic $(17,19)$. These findings are consistent with prior reports that documented high sedative and neuromuscular blockade use in COVID patients(20, 22, 36-39). Further, our findings highlight the static nature in the approach to sedation in the early deep sedation group: 1) > 50\% experienced deep sedation throughout the first week of mechanical ventilation; and 2) $33 \%$ were deeply sedated until death. While not formally measured in this study, these results further suggest low adherence to the ABCDEF bundle, congruent with a prior international point prevalence study on ICU patients with COVID (40).

Another important finding involves the sedation observed in non-COVID patients. Given the significant changes in supportive care observed during the onset of the COVID pandemic, it is reasonable to hypothesize that the care of non-COVID patients would have been altered as well. However, when 
compared to prior work, patients in the non-COVID group experienced sedation management, early deep sedation, and clinical outcomes similar to that seen in the pre-COVID era (17). This suggests that the observed changes in the standards of critical care were isolated to COVID patients, and further highlights the importance of continued assessments into protocol-driven supportive care in this cohort.

This work has several important limitations. This is one of the first studies examining the impact of sedation depth on clinical outcomes during the COVID pandemic, yet it is relatively small and therefore prone to bias. As a two-center study, it is possible that these data are not truly representative and lack external validity. All data were obtained retrospectively and therefore subject to potential inaccuracies in routine documentation. The study design can only inform on association and not causation, and the ability to control for confounding is limited. Deep sedation, and therefore the possible the need for it, overlapped with COVID status, and may also have been a marker of illness severity and the presence of ARDS. Our results are consistent with prior literature regarding the impact of early deep sedation on outcomes, and the association between deep sedation and mortality remained strong after adjusting for SOFA (which includes oxygenation). While this is encouraging and lends face validity, however the relationship between early deep sedation and disease severity is difficult to truly separate through statistical methods. As such, these results should be viewed as hypothesis-generating. These data were collected during the first six months of the COVID pandemic, and therefore may not reflect rapidly evolving COVID era sedation practices. However, this work highlights the importance of adhering to proven ICU principles and are informative for the potential of persistent COVID-19 or future viral pandemics. Finally, depressed mental status and deeper sedation levels may have been secondary to COVID and/or structural lesions, as opposed to sedation management (41). Since no imaging data were collected for this study, this remains a potential confounder.

\section{Conclusion}

The management of sedation for mechanically ventilated patients in the ICU has been impacted by the COVID pandemic. Early deep sedation is common, especially among COVID-19 patients, and independently associated with worse clinical outcomes. A protocol-driven approach to sedation, targeting light sedation as early as possible, should continue to remain the default approach.

\section{Abbreviations}

ARDS

acute respiratory distress syndrome

COVID

coronavirus disease

ED

emergency department

$\mathrm{FiO} 2$

fraction of inspired oxygen

Page 18/25 
HRPO

Human Research Protection Office

ICU

intensive care unit

IRB

Institutional Review Board

OR

operating room

$\mathrm{PaO} 2$

partial pressure of arterial oxygenation

RASS

Richmond Agitation-Sedation Scale

SAS

Sedation-Agitation Scale

SOFA

sequential organ failure assessment

STROBE

Strengthening Reporting of Observational Studies in Epidemiology

\section{Declarations}

\section{Competing Interests}

All authors report no competing interests

\section{Funding Information}

MHK is supported by the Barnes-Jewish Hospital Foundation. BMF is supported by the National Heart, Lung, and Blood Institute of the National Institutes of Health (NIH) under award number R34HL150404.

The content is solely the responsibility of the authors and does not necessarily represent the official views of the NIH. Funders played no role in the following features of the study: study design, data collection, data management, data analysis, data interpretation, writing of the manuscript or decision to submit the manuscript for publication.

\section{Notification of prior abstract publication/presentation}

Partial results from this study have been accepted as an abstract for presentation at the Society of Critical Care Medicine (April, 2022) annual congress.

Ethical approval: The Institutional Review Board and Human Research Protection Office at each site approved the study with waiver of informed consent prior to study initiation (IRB \# 202009119 and 202009604) 
Consent for publication: Study was performed under a waiver of informed consent approved by the Institutional Review Board and Human Resources Protection Office

Data Availability Statement: The datasets used and/or analyzed during the current study are available from the corresponding author on reasonable request.

Financial/nonfinancial disclosures: All authors have nothing to declare.

Role of the sponsors: Funders played no role in the following features of the study: study design, data collection, data management, data analysis, data interpretation, writing of the manuscript or decision to submit the manuscript for publication.

Other contributions: None to declare.

Guarantor statement: BMF takes responsibility for the content of the manuscript as a whole.

\section{Author contributions:}

RJS: conception and study design, acquisition of data, analysis and interpretation of data, drafting and revising the manuscript

EME: conception and study design, acquisition of data, analysis and interpretation of data, drafting and revising the manuscript

MJP: acquisition of data, analysis and interpretation of data, drafting and revising the manuscript RDP: study design, acquisition of data, analysis and interpretation of data, drafting and revising the manuscript

HME: acquisition of data, analysis and interpretation of data, drafting and revising the manuscript MW: acquisition of data, analysis and interpretation of data, drafting and revising the manuscript $\mathrm{HH}$ : acquisition of data, analysis and interpretation of data, drafting and revising the manuscript JAM: acquisition of data, analysis and interpretation of data, drafting and revising the manuscript NB: acquisition of data, analysis and interpretation of data, drafting and revising the manuscript BWR: conception and study design, acquisition of data, analysis and interpretation of data, drafting and revising the manuscript

MHK: conception, analysis and interpretation of data, drafting and revising the manuscript

NMM: conception and study design, acquisition of data, analysis and interpretation of data, drafting and revising the manuscript 
BMF: conception and study design, acquisition of data, analysis and interpretation of data, drafting and revising the manuscript

\section{References}

1. Harhay MO, Wagner J, Ratcliffe SJ, Bronheim RS, Gopal A, Green S, et al. Outcomes and statistical power in adult critical care randomized trials. American journal of respiratory and critical care medicine. 2014;189(12):1469-78.

2. Morris PE, Goad A, Thompson C, Taylor K, Harry B, Passmore L, et al. Early intensive care unit mobility therapy in the treatment of acute respiratory failure. Critical care medicine. 2008;36(8):223843.

3. Pronovost P, Needham D, Berenholtz S, Sinopoli D, Chu H, Cosgrove S, et al. An intervention to decrease catheter-related bloodstream infections in the ICU. New England journal of medicine. 2006;355(26):2725-32.

4. Network ARDS, Wheeler A, Bernard G, Thompson B, Hayden D, DeBoisblanc B. Comparison of two fluid-management strategies in acute lung injury. N Engl j Med. 2006;354(24):2564-75.

5. Ventilation with lower tidal volumes as compared with traditional tidal volumes for acute lung injury and the acute respiratory distress syndrome. The Acute Respiratory Distress Syndrome Network. N Engl J Med. 2000;342(18):1301-8.

6. Girard TD, Kress JP, Fuchs BD, Thomason JW, Schweickert WD, Pun BT, et al. Efficacy and safety of a paired sedation and ventilator weaning protocol for mechanically ventilated patients in intensive care (Awakening and Breathing Controlled trial): a randomised controlled trial. The Lancet. 2008;371(9607):126-34.

7. Kollef MH, Levy NT, Ahrens TS, Schaiff R, Prentice D, Sherman G. The use of continuous iv sedation is associated with prolongation of mechanical ventilation. Chest. 1998;114(2):541-8.

8. Kress JP, O'Connor MF, Pohlman AS, Olson D, Lavoie A, Toledano A, et al. Sedation of critically ill patients during mechanical ventilation. A comparison of propofol and midazolam. American journal of respiratory and critical care medicine. 1996;153(3):1012-8.

9. Kress JP, Pohlman AS, O'Connor MF, Hall JB. Daily interruption of sedative infusions in critically ill patients undergoing mechanical ventilation. New England Journal of Medicine. 2000;342(20):14717.

10. Mehta S, Burry L, Cook D, Fergusson D, Steinberg M, Granton J, et al. Daily sedation interruption in mechanically ventilated critically ill patients cared for with a sedation protocol: a randomized controlled trial. Jama. 2012;308(19):1985-92.

11. Quenot J-P, Ladoire S, Devoucoux F, Doise J-M, Cailliod R, Cunin N, et al. Effect of a nurseimplemented sedation protocol on the incidence of ventilator-associated pneumonia. Critical care medicine. 2007;35(9):2031-6. 
12. Reade MC, Finfer S. Sedation and delirium in the intensive care unit. New England Journal of Medicine. 2014;370(5):444-54.

13. Shehabi Y. The Golden Hours of ICU Sedation: The Clock Is Ticking. Critical care medicine. 2018;46(3):490-1.

14. Shehabi Y, Bellomo R, Kadiman S, Ti LK, Howe B, Reade MC, et al. Sedation Intensity in the First 48 Hours of Mechanical Ventilation and 180-Day Mortality: A Multinational Prospective Longitudinal Cohort Study. Critical care medicine. 2018;46(6):850-9.

15. Shehabi Y, Bellomo R, Reade MC, Bailey M, Bass F, Howe B, et al. Early intensive care sedation predicts long-term mortality in ventilated critically ill patients. American journal of respiratory and critical care medicine. 2012;186(8):724-31.

16. Shehabi Y, Chan L, Kadiman S, Alias A, Ismail WN, Tan MATI, et al. Sedation depth and long-term mortality in mechanically ventilated critically ill adults: a prospective longitudinal multicentre cohort study. Intensive care medicine. 2013;39(5):910-8.

17. Fuller BM, Roberts BW, Mohr NM, Knight IV WA, Adeoye O, Pappal RD, et al. The ED-SED Study: A Multicenter, Prospective Cohort Study of Practice Patterns and Clinical Outcomes Associated With Emergency Department SEDation for Mechanically Ventilated Patients. Critical Care Medicine. 2019;47(11):1539-48.

18. Stephens RJ, Ablordeppey E, Drewry AM, Palmer C, Wessman BT, Mohr NM, et al. Analgosedation practices and the impact of sedation depth on clinical outcomes among patients requiring mechanical ventilation in the ED: a cohort study. Chest. 2017;152(5):963-71. PMCID: PMC5812748.

19. Stephens RJ, Dettmer MR, Roberts BW, Ablordeppey E, Fowler SA, Kollef MH, et al. Practice Patterns and Outcomes Associated With Early Sedation Depth in Mechanically Ventilated Patients: A Systematic Review and Meta-Analysis. Critical Care Medicine. 2017;46 (3):471-9. PMCID: PMC5825247.

20. Wongtangman K, Santer P, Wachtendorf LJ, Azimaraghi O, Kassis EB, Teja B, et al. Association of Sedation, Coma, and In-Hospital Mortality in Mechanically Ventilated Patients With Coronavirus Disease 2019-Related Acute Respiratory Distress Syndrome: A Retrospective Cohort Study. Critical Care Medicine. 2021.

21. [Pubmed search for "COVID-19" and "COVID- AND sedation"].

22. Pun BT, Badenes R, La Calle GH, Orun OM, Chen W, Raman R, et al. Prevalence and risk factors for delirium in critically ill patients with COVID-19 (COVID-D): a multicentre cohort study. The Lancet Respiratory medicine. 2021;9(3):239-50.

23. Zampieri FG, Bastos LSL, Soares M, Salluh JI, Bozza FA. The association of the COVID-19 pandemic and short-term outcomes of non-COVID-19 critically ill patients: an observational cohort study in Brazilian ICUs. Intensive Care Med. 2021;47(12):1440-9.

24. von Elm E, Altman, DG, Egger, M, Pocock, SJ, Gotzsche, PC, Vandenbroucke, JP. The Strengthening the Reporting of Observational Studies in Epidemiology (STROBE) Statement: Guidelines for Reporting Observational Studies. Annals of Internal Medicine. 2007;147(8):573-7. 
25. Harris PA, Taylor R, Minor BL, Elliott V, Fernandez M, O'Neal L, et al. The REDCap consortium: Building an international community of software platform partners. Journal of biomedical informatics. 2019;95:103208.

26. Harris PA, Taylor R, Thielke R, Payne J, Gonzalez N, Conde JG. Research electronic data capture (REDCap) - a metadata-driven methodology and workflow process for providing translational research informatics support. Journal of biomedical informatics. 2009;42(2):377-81.

27. Vincent J, Moreno R, Takala J, Willatts S, De Mendonça A, Bruining H, et al. The SOFA (Sepsis-related Organ Failure Assessment) score to describe organ dysfunction/failure. Intensive care medicine. 1996;22:707-10.

28. Vincent J-L, De Mendonça A, Cantraine F, Moreno R, Takala J, Suter PM, et al. Use of the SOFA score to assess the incidence of organ dysfunction/failure in intensive care units: results of a multicenter, prospective study. Critical care medicine. 1998;26(11):1793-800.

29. Tanaka LMS, Azevedo LCP, Park M, Schettino G, Nassar AP, Réa-Neto A, et al. Early sedation and clinical outcomes of mechanically ventilated patients: a prospective multicenter cohort study. Critical Care. 2014;18(4):1.

30. Maslove DM, Leisman DE. Causal inference from observational data: New guidance from pulmonary, critical care, and sleep journals. Critical Care Medicine. 2019;47(1):1-2.

31. Fuller BM, Ferguson IT, Mohr NM, Drewry AM, Palmer C, Wessman BT, et al. Lung-protective ventilation initiated in the emergency department (LOV-ED): a quasi-experimental, before-after trial. Annals of Emergency Medicine. 2017;70(3):406-18. PMCID: PMC5573637.

32. Fuller BM, Ferguson IT, Mohr NM, Drewry AM, Palmer C, Wessman BT, et al. A quasi-experimental, before-after trial examining the impact of an emergency department mechanical ventilator protocol on clinical outcomes and lung-protective ventilation in acute respiratory distress syndrome. Critical Care Medicine. 2017;45(4):645-52. PMCID: PMC5350028.

33. Fuller BM, Mohr NM, Miller CN, Deitchman AR, Levine BJ, Castagno N, et al. Mechanical Ventilation and ARDS in the ED: A Multicenter, Observational, Prospective, Cross-sectional Study. Chest. 2015;148(2):365-74. PMCID: PMC4524326.

34. Pappal RD, Roberts BW, Mohr NM, Ablordeppey E, Wessman BT, Drewry AM, et al. The EDAWARENESS Study: A Prospective, Observational Cohort Study of Awareness With Paralysis in Mechanically Ventilated Patients Admitted From the Emergency Department. Annals of Emergency Medicine. 2021;77(5):532-44.

35. Devlin JW, Pandharipande PP. Do Our Sedation Practices Contribute to Increased Mortality in Coronavirus Disease 2019-Related Acute Respiratory Distress Syndrome? Critical Care Medicine. 2021;49(9):1579-82.

36. Tapaskar N, Colon Hidalgo D, Koo G, Shingada K, Rao S, Rodriguez R, et al. Sedation Usage in COVID19 Acute Respiratory Distress Syndrome: A Multicenter Study. Annals of Pharmacotherapy. 2021:10600280211021925. 
37. Flinspach AN, Booke H, Zacharowski K, Balaban Ü, Herrmann E, Adam EH. High sedation needs of critically ill COVID-19 ARDS patients-A monocentric observational study. PloS one. 2021;16(7):e0253778.

38. Khan SH, Lindroth H, Perkins AJ, Jamil Y, Wang S, Roberts S, et al. Delirium Incidence, Duration, and Severity in Critically III Patients With Coronavirus Disease 2019. Critical care explorations. 2020;2(12).

39. Balakrishna A, Walsh EC, Hamidi A, Berg S, Austin D, Pino RM, et al. An examination of sedation requirements and practices for mechanically ventilated critically ill patients with COVID-19. American Journal of Health-System Pharmacy: AJHP. 2021.

40. Liu K, Nakamura K, Katsukawa H, Elhadi M, Nydahl P, Ely EW, et al. ABCDEF Bundle and supportive ICU practices for patients with coronavirus disease 2019 infection: An international point prevalence study. Critical care explorations. 2021;3(3).

41. Kremer S, Lersy F, de Sèze J, Ferré J-C, Maamar A, Carsin-Nicol B, et al. Brain MRI findings in severe COVID-19: a retrospective observational study. Radiology. 2020;297(2):E242-E51.

\section{Figures}

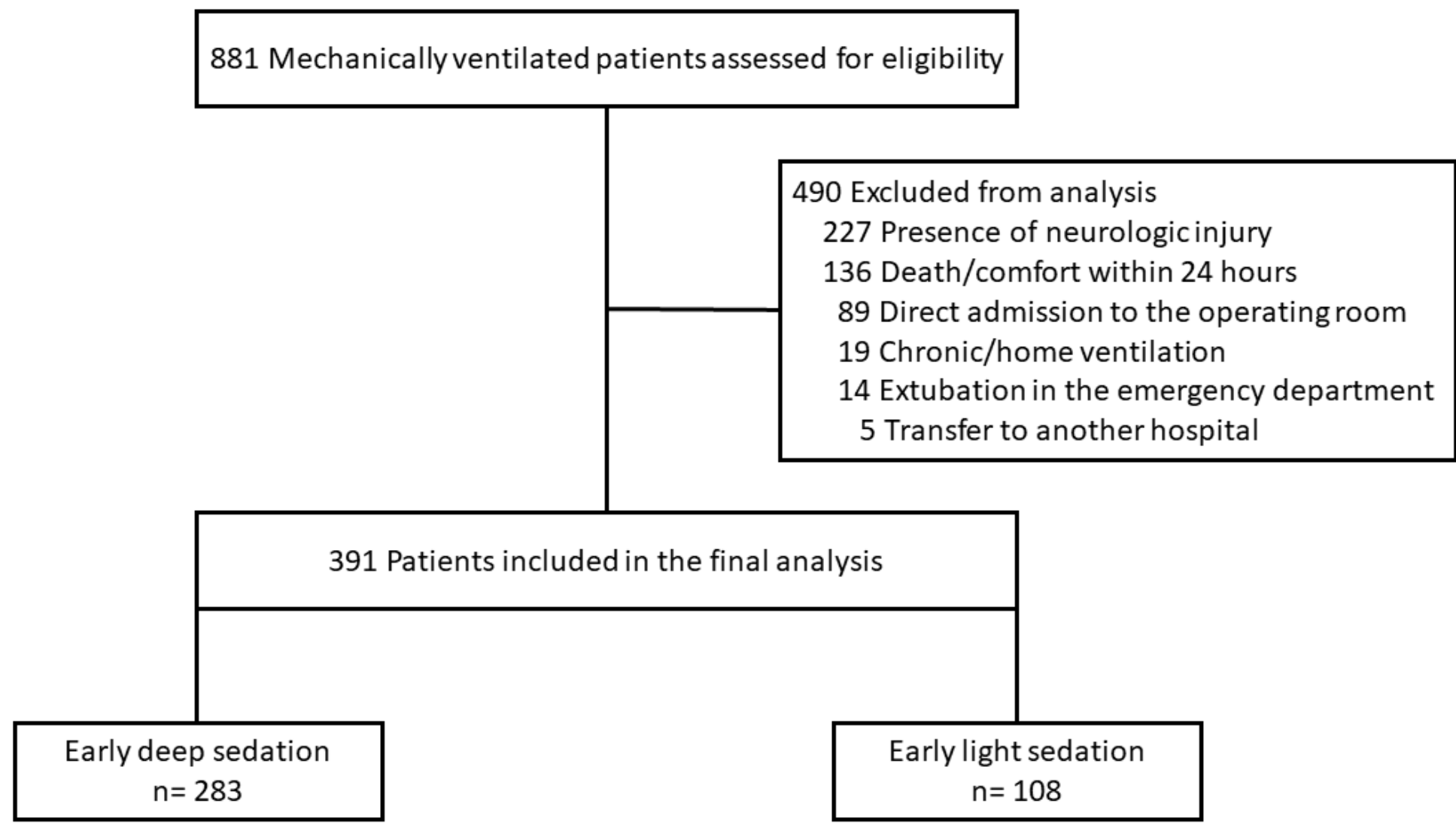

Figure 1

Study flow diagram 


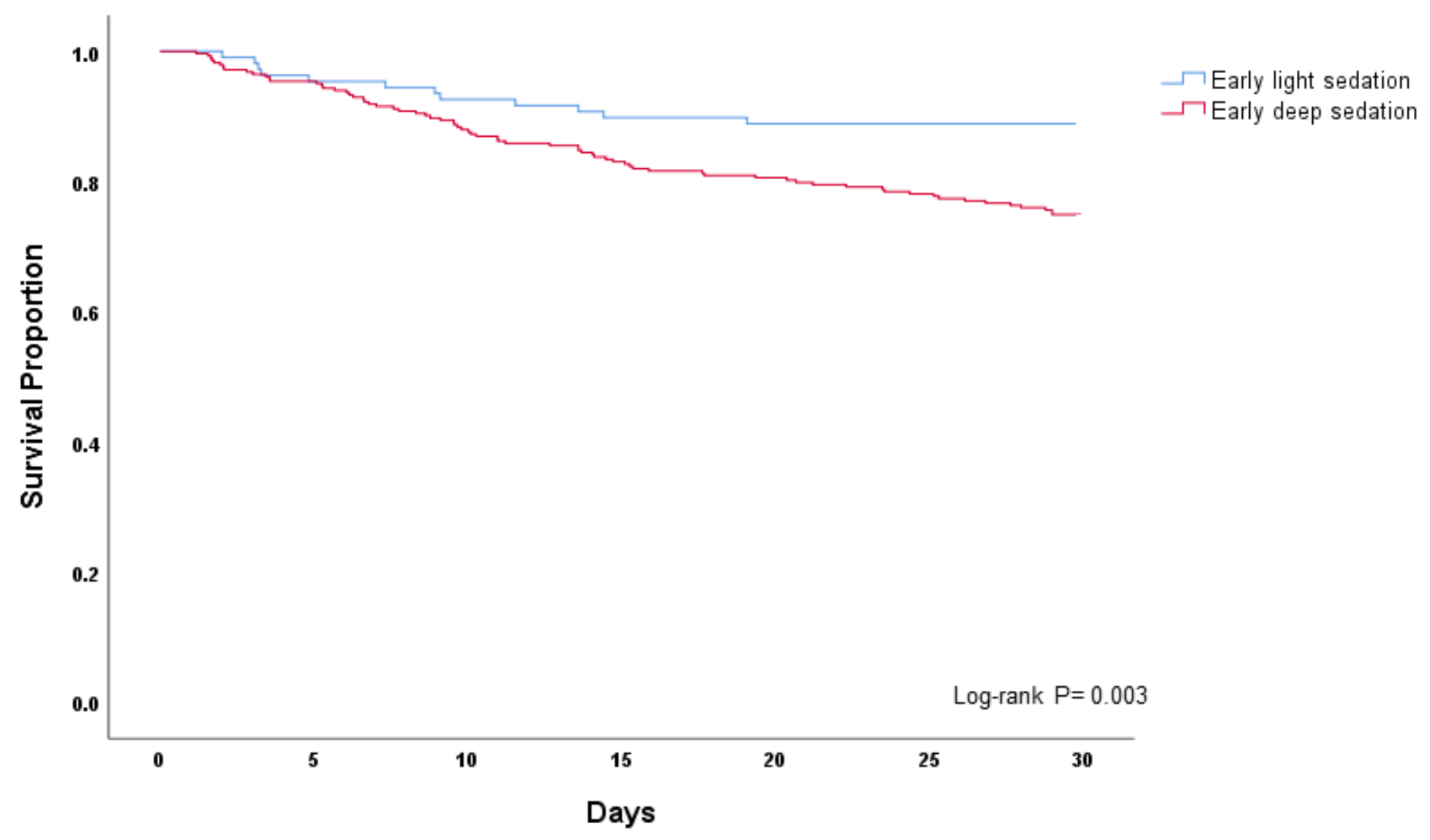

Figure 2

Kaplan-Meier survival curve between the early deep and light sedation groups

\section{Supplementary Files}

This is a list of supplementary files associated with this preprint. Click to download.

- AdditionalFile1STROBEChecklistCOVIDSED.doc

- AdditionalTable1 IntubationmedsCOVIDSEDSedationdepthstatus.docx

- AdditionalTable2SedationvariablesintheEDCOVIDSEDSedationdepthstatus.docx

- AdditionalTable3BaselinecharacteristicsCOVIDSEDCOVIDStatus.doc

- AdditionalTable4SedationvariablesintheEDCOVIDSEDCOVIDStatus.docx

- AdditionalTable5Multivariablelogisticregressionmodelformortality.docx

- AdditionalTable6ClinicaloutcomesandregressionanalysisCOVIDSEDCOVIDStatus.doc 